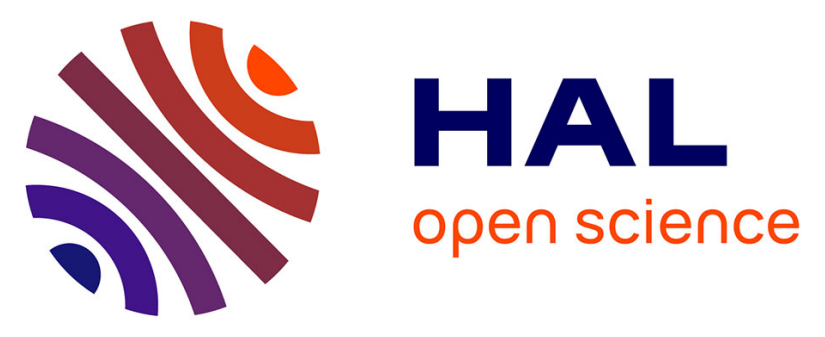

\title{
Influence of barrier layer indium on efficiency and wavelength of InGaN multiple quantum well (MQW) with and without semi-bulk InGaN buffer for blue to green regime emission
}

Saiful Alam, Suresh Sundaram, Xin Li, Miryam Jamroz, Youssef El Gmili, Ivan Robin, Paul B Voss, Jean-Paul Salvestrini, Abdallah Ougazzaden

\section{To cite this version:}

Saiful Alam, Suresh Sundaram, Xin Li, Miryam Jamroz, Youssef El Gmili, et al.. Influence of barrier layer indium on efficiency and wavelength of InGaN multiple quantum well (MQW) with and without semi-bulk InGaN buffer for blue to green regime emission. physica status solidi (a), 2017, 214 (8), pp.1600868. 10.1002/pssa.201600868 . hal-01830966

\author{
HAL Id: hal-01830966 \\ https://hal.science/hal-01830966
}

Submitted on 19 Jan 2022

HAL is a multi-disciplinary open access archive for the deposit and dissemination of scientific research documents, whether they are published or not. The documents may come from teaching and research institutions in France or abroad, or from public or private research centers.
L'archive ouverte pluridisciplinaire HAL, est destinée au dépôt et à la diffusion de documents scientifiques de niveau recherche, publiés ou non, émanant des établissements d'enseignement et de recherche français ou étrangers, des laboratoires publics ou privés.

\section{(ㅇ)(1) $\$$}

Distributed under a Creative Commons Attribution - NonCommerciall 4.0 International 


\title{
Influence of barrier layer indium on efficiency and wavelength of InGaN multiple quantum well (MQW) with and without semi-bulk InGaN buffer for blue to green regime emission
}

\author{
Saiful Alam ${ }^{1,2,3}$, Suresh Sundaram ${ }^{2}$, Xin li ${ }^{2}$, Miryam E. Jamroz ${ }^{3}$, Youssef El Gmili², Ivan C. Robin ${ }^{3}$, Paul L. Voss ${ }^{1,2}$, \\ Jean-Paul Salvestrini ${ }^{2,4}$, and Abdallah Ougazzaden ${ }^{*, 1,2}$ \\ 1 School of Electrical and Computer Engineering, Georgia Institute of Technology, Atlanta, Georgia 30332, USA \\ 2 Georgia Tech-CNRS, UMI 2958, 57070 Metz, France \\ 3 CEA-LETI, Minatec Campus, F-38054 Grenoble, France \\ 4 LMOPS, University of Lorraine, EA4423, 57070 Metz, France
}

\begin{abstract}
The effect of indium (In) in the barrier of InGaN/GaN multiple quantum well (MQW) has been studied for MQWs with and without semi-bulk InGaN buffer. From simulation, the optimum In content in the barrier with $3-5 \mathrm{~nm}$ width is $5-7 \%$ to get the optimal material quality and internal quantum efficiency (IQE) of $\sim 65 \%$ for $450-480 \mathrm{~nm}$ emission range. Simulation shows a reduction of the potential barrier due to band flattening, a more homogeneous distribution of electrons and holes in the active region and subsequently, a more radiative recombination rate with $\mathrm{InGaN}$ as barrier layer. Both cathodoluminescence (CL) and
\end{abstract}

photoluminescence (PL) experimental results show a blueshift of emission wavelength along with an enhancement in the emission intensity when GaN barrier is replaced with InGaN barrier, for a MQW structure both with and without the semi-bulk InGaN buffer. We attribute this blue shift to the reduced polarization mismatch and increased effective bandgap. This InGaN barrier-related improvement in IQE and efficiency droop could be useful for the realization of longer wavelength "green-gap" range LEDs where poor IQE and efficiency droop are more prominent due to high indium (In) in the active region.

Keywords III-nitride semiconductors, barrier layer, InGaN, light-emitting diodes, MOVPE, multiple quantum well

1 Introduction InGaN/GaN-based visible light emitting diodes (LEDs) are commercially successful and play a dominant role in solid state lighting [1]. However, at green emission wavelengths, the light emission efficiency of the devices grown in the commonly used + c-direction of GaN decreases with increasing indium (In) content in the active region [1]. Primarily, this is attributed to the increased piezoelectric field in the InGaN/GaN quantum well (QW) under biaxial compressive strain that eventually leads to local separation of electrons and holes within the QWs, and consequently decreases the emission efficiency [1]. Even though growing stable semi-polar or non-polar planes [2] by non-conventional growth has received much attention for minimizing the polarization problem, good crystal quality and processes that are mask-free and result in fewer processing steps make (0001) direction growth still commercially promising. Apart from the polarization problem and the efficiency droop at high current density [3], one major problem in InGaN/GaN MQW is the potential barrier that causes the hole injection difficulty because of the comparatively lower mobility of the hole. Furthermore, commonly used $\mathrm{AlGaN}$ electron blocking layer (EBL) makes the hole transport more difficult and hence, most of the holes are gathered in the last QW (closest to the pside) [3, 4]. This, along with the bend bending, causes electron leakage to the $\mathrm{p}-\mathrm{GaN}$ [5] instead of recombination 
in the active region, which results in an efficiency droop. To overcome these challenges, recent reports have proposed sloped triangular barrier [6,7], step graded AlGaN EBL [8, 9], graded $\mathrm{InGaN} / \mathrm{GaN}$ superlattice interlayer [10], trapezoidal quantum well [11], InGaN/InGaN MQW [4, 12], graded InGaN and alternating InGaN/GaN barriers [13]. The InGaN/InGaN MQWs have been studied in previous theoretical studies by simulation $[4,5,13]$ and in some experimental studies [13] that focused mainly on the improvement of polarization matching, reduction of potential barrier, improved optical output power, and improved IQE. However, the experimental study of the effect of InGaN barrier on the emission wavelength in combination with theoretical simulation, and the comparative study of InGaN barrier for MQWs with and without InGaN semi-bulk (SB) buffer, are still necessary. In this article, we present the simulation study of the advantage of InGaN barrier. Moreover, the effect of InGaN barrier on the emission wavelength and the intensity of MQW structure with and without InGaN semi-bulk buffer, particularly for longer wavelength emission in the "green-gap" regime, has been studied. This study could be useful to realize LEDs with the "green-gap" regime wavelength emission with high IQE and low droop.

2 Experimental The MQW samples were grown by a T-shape horizontal flow low-pressure metal organic vapor phase epitaxy (MOVPE) reactor on $3.5 \mu \mathrm{m} \mathrm{n}$-GaN templates (STN) on (0001) sapphire substrate [14]. The growth temperature was $795^{\circ} \mathrm{C}$ for InGaN growth and $1000^{\circ} \mathrm{C}$ for $\mathrm{GaN}$ growth. The reactor pressure was 100 Torr. Nitrogen $\left(\mathrm{N}_{2}\right)$, hydrogen $\left(\mathrm{H}_{2}\right)$ were used as carrier gases and trimethylgallium (TMGa), trimethylindium (TMIn), and ammonia $\left(\mathrm{NH}_{3}\right)$ were employed as precursor sources for $\mathrm{Ga}$, In and elemental $\mathrm{N}$, respectively. Calibrated TMIn precursor flow and growth temperature were maintained to achieve the desired indium (In) incorporation in InGaN layers. On the $\mathrm{GaN}$ STN template, first $1 \mu \mathrm{m}$ of $\mathrm{n}-\mathrm{GaN}$ was grown followed by MQWs with a 3-period of $5 \mathrm{~nm}$ barrier and a $3 \mathrm{~nm} \mathrm{In} \operatorname{In}_{0.18} \mathrm{Ga}_{0.82} \mathrm{~N}$ well for samples $\mathrm{A}$ and $\mathrm{B}$. The barriers were $\mathrm{GaN}$ for sample $\mathrm{A}$ and $\mathrm{In}_{0.05} \mathrm{Ga}_{0.85} \mathrm{~N}$ for sample $\mathrm{B}$. Samples $\mathrm{C}$ and D had the same structure with $\mathrm{GaN}$ and $\mathrm{In}_{0.05} \mathrm{Ga}_{0.95} \mathrm{~N}$ barrier, respectively, except that the MQWs were grown on a $\sim 70 \mathrm{~nm}$ thick (4-period, $15 \mathrm{~nm}$ $\mathrm{In}_{0.05} \mathrm{Ga}_{0.95} \mathrm{~N}$ with $2 \mathrm{~nm} \mathrm{GaN}$ interlayers in between two successive InGaN layers) semi-bulk (SB) InGaN buffer layer. The structures are shown schematically in Fig. 1. High-resolution X-ray diffraction (XRD) measurements were performed in a Panalytical X'pert Pro MRD system with $\mathrm{Cu}-\mathrm{K}_{\alpha}$ radiation [15]. The thicknesses of layers were determined from an in situ reflectometry and confirmed by transmission electron microscopy (TEM). The surface morphology was observed by scanning electron microscopy (SEM) and atomic force microscopy (AFM). Optical emission properties of InGaN structures were investigated by both cathodoluminescence (CL) and photoluminescence (PL) measurements. Room and low temperature CL investigations were performed in a digital scanning electron microscope [15]. For low temperature CL measurement, the samples were cooled inside the SEM with $\mathrm{LN}_{2}$-cooled $\mathrm{N}_{2}$ vapor. Each recorded spectrum was mathematically processed, fitted using Gauss functions and plotted. Low temperature photoluminescence was carried out at $15 \mathrm{~K}$ in a helium cryostat using a $375 \mathrm{~nm}$ laser as an excitation source. The excitation power was $10 \mathrm{~mW}$ and the beam area was $\sim 100 \mu \mathrm{m}$. The MQW structures were simulated by commercial Silvaco TCAD software, where self-consistent band structures and wave functions were obtained by solving the Schrödinger equation for electrons, Hamiltonian for holes, and Poisson's equation iteratively [16]. The driftdiffusion k.p model was used, interface and surface charge were considered, and material parameters were taken from Bernardini et al. [17]. The bias was $50 \mathrm{~A}$ and upto $4 \mathrm{~V}$.

3 Results and discussion The optimum indium (In) content in the barrier was obtained from the simulation.

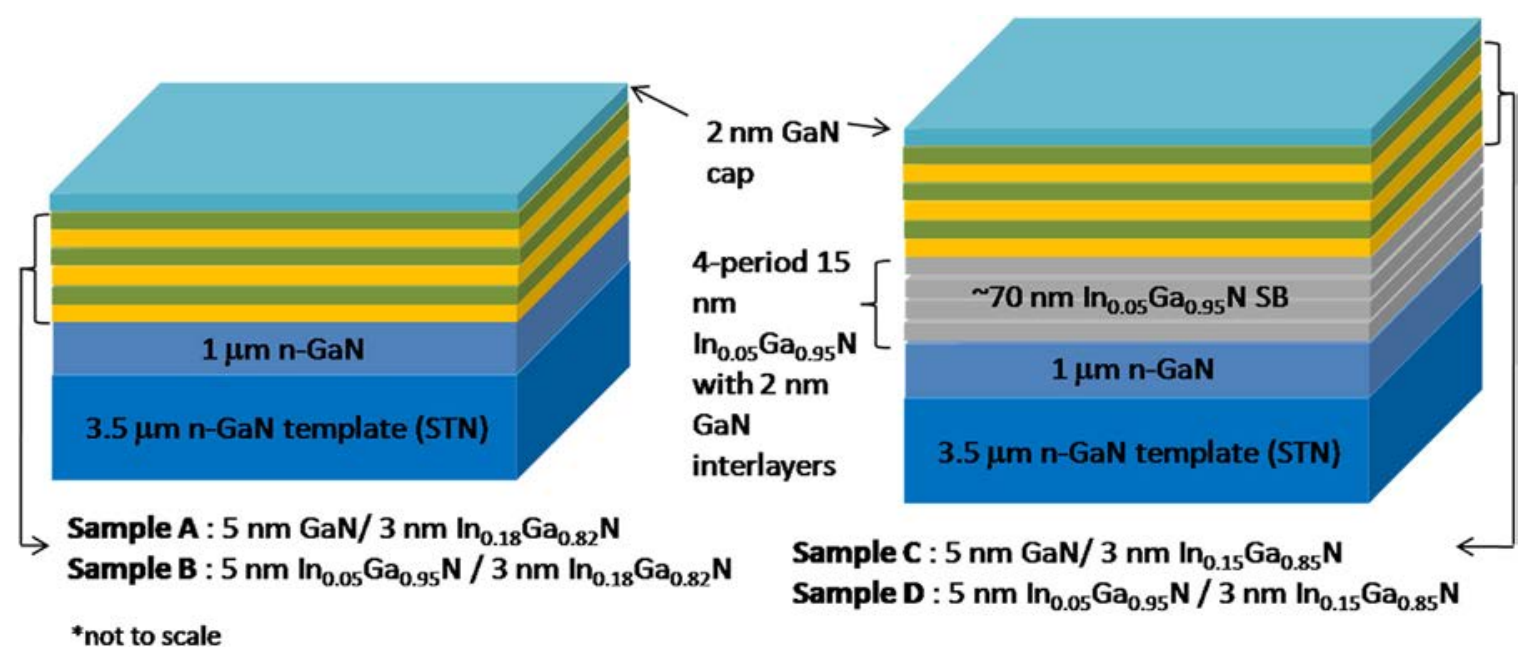

Figure 1 Structure schematic of the different samples used in this study (left: samples A and B; right: samples C and D). 

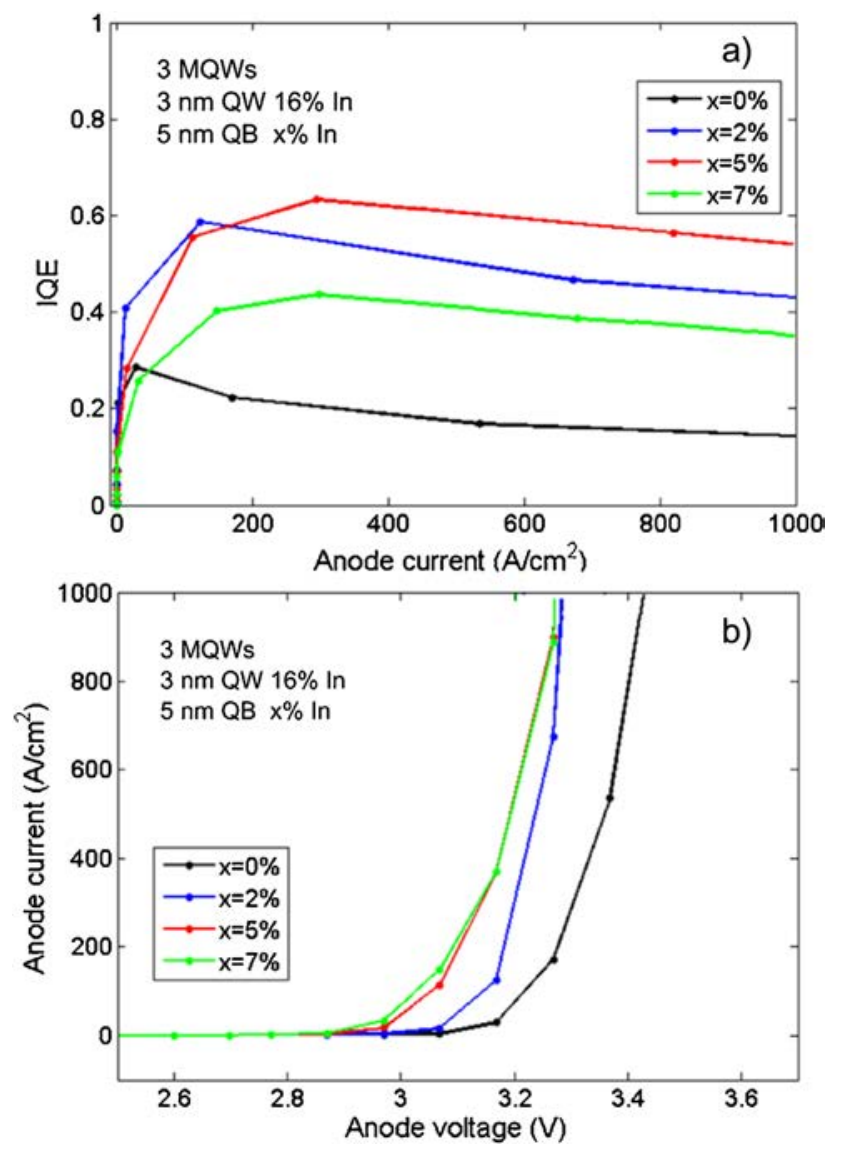

Figure 2 Effect of different In contents in the barrier: IQE (a) and $I-V$ curves (b).

Figure 2 shows the room temperature internal quantum efficiency (IQE) and current-voltage $(I-V)$ curves for different In content in a 3-period MQW with a $3 \mathrm{~nm}$ well and a $5 \mathrm{~nm}$ barrier.

The best IQE is observed for 5\% In in the barrier, whereas, In content higher than this results in a deterioration of the IQE modeled by increased SRH and Auger recombinations. This is consistent with experimental result where In content higher than $5 \%$ causes worse crystal quality leading to more non-radiative recombination centers [18]. The simulation was performed for different number of quantum wells for sample A with $\mathrm{GaN}$ barrier and sample B with $\mathrm{In}_{0.05} \mathrm{Ga}_{0.95} \mathrm{~N}$ barrier. The comparison of IQE is shown in Fig. 3 and $I-V$ characteristics in Fig. 4.

InGaN barrier gives $\sim 170 \%$ improvement of IQE for 3 QWs at $200 \mathrm{~A} \mathrm{~cm}^{-2}$. Since a sufficient active region volume is required to get the maximum radiative output, 3 or 5 is the optimum number of QWs to get the maximum efficiency. The turn on voltage has also improved for InGaN barrier (Fig. 4) and is less than $3 \mathrm{~V}$ for optimal number of QWs. This improvement is attributed to an improved hole injection due to the reduced potential barrier [13]. To further investigate the improved IQE for InGaN barriers, the calculated radiative recombination rates were plotted and compared.
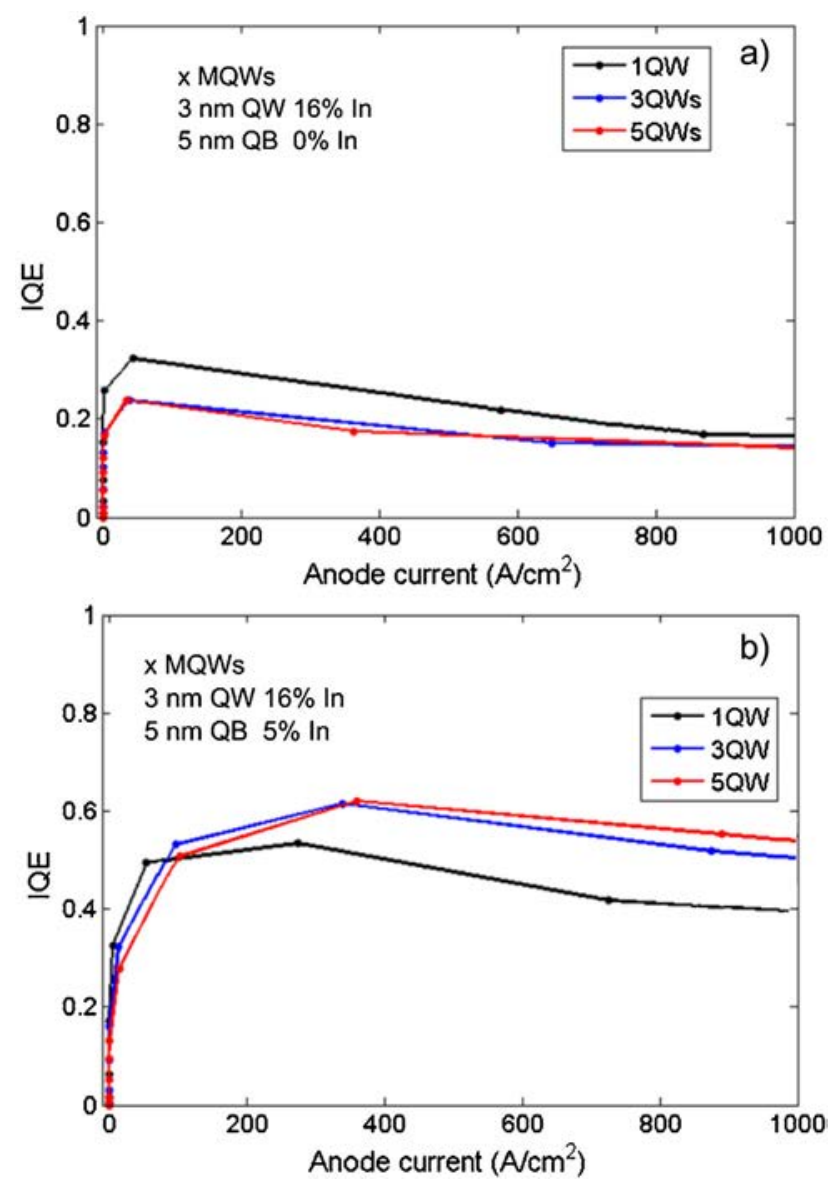

Figure 3 Simulated IQE comparison of sample A with GaN barrier (a) and sample B with $\operatorname{In}_{0.05} \mathrm{Ga}_{0.95} \mathrm{~N}$ barrier (b).

Figure 5 shows the radiative recombination rate for samples $A$ and $B$. With InGaN barriers, radiative recombination takes place in all the wells rather than only in the last well and the total radiative recombination rate increases by $\sim 5.8 \times 10^{26} \mathrm{~cm}^{-3} \mathrm{~s}^{-1}$.

Figure 6 shows the electron and hole concentrations for GaN barrier (Fig. 6(a)) and for InGaN barrier (Fig. 6(b)) in the otherwise same MQWs. It can be easily observed that the electrons are more uniformly distributed in all the wells and the total electron concentration has also increased for InGaN barrier. Moreover, though in the MQW with GaN barrier, holes are only gathered in the last QW (closest to the p side) due to low mobility; in the MQW structure with InGaN barrier, due to low potential barrier height, holes are evenly distributed in all the wells (Fig. 6(b)). In the simulated band diagram, shown in Fig. 6(c), the band bending is lower for the InGaN barrier and this reduced energy barrier for electrons and holes is the cause for a more uniform carrier distribution in wells. This homogeneous distribution results in more overlapping of the electron and hole wave functions and consequently in more radiative recombination.

However, the increased effective bandgap should give a shorter wavelength emission which could be a drawback to 

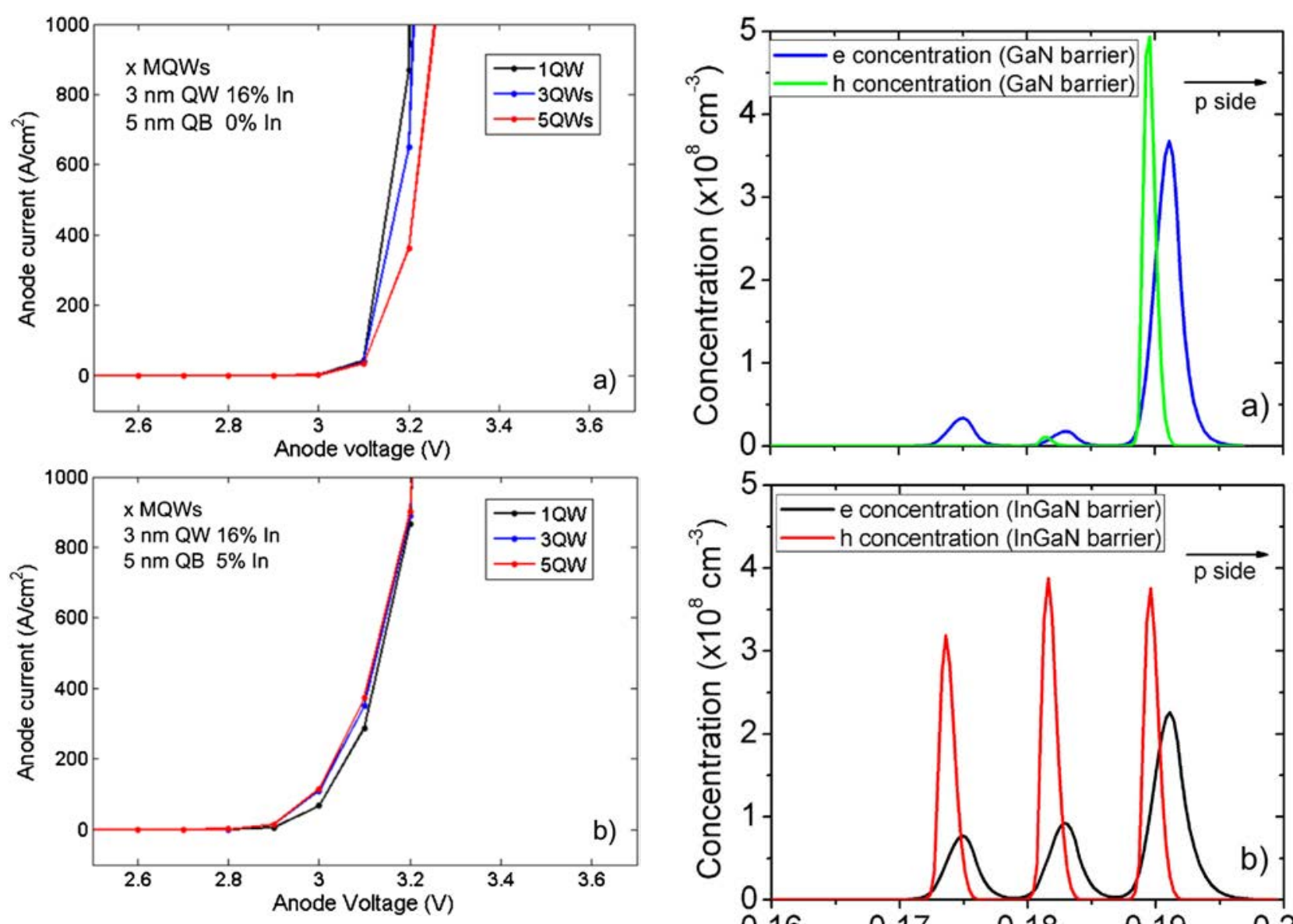

Figure 4 Simulated $I-V$ curves for sample with GaN barrier (a) and $\mathrm{In}_{0.05} \mathrm{Ga}_{0.95} \mathrm{~N}$ barrier (b).

get longer wavelength emission in the "green-gap" regime. This blue-shift can be counteracted by the red-shift caused by growing the MQW on a semi-bulk InGaN buffer layer which is discussed later.

We investigated the simulation result experimentally. Studies of the surface morphology of the sample with GaN
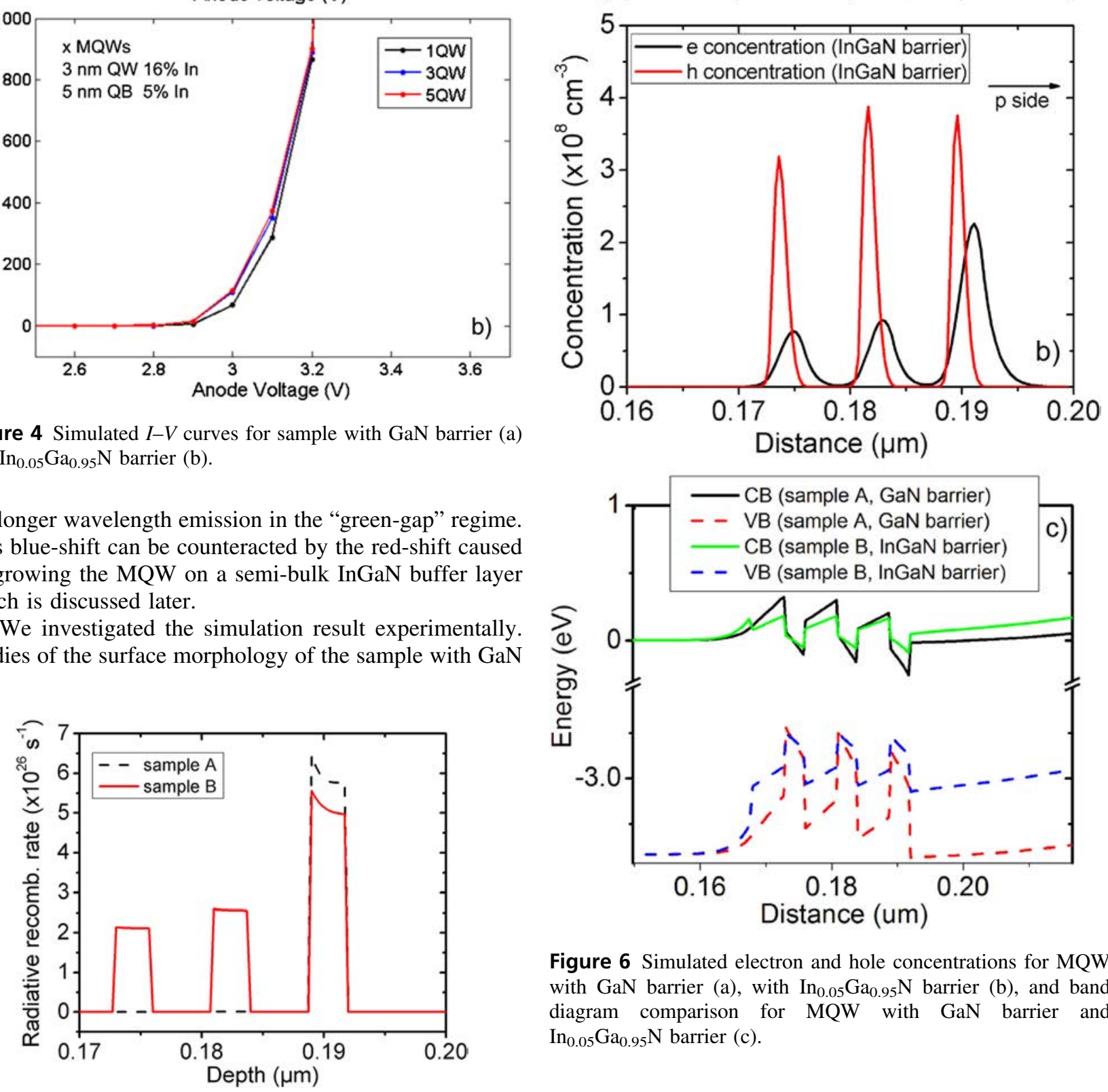

Figure 6 Simulated electron and hole concentrations for MQW with $\mathrm{GaN}$ barrier (a), with $\mathrm{In}_{0.05} \mathrm{Ga}_{0.95} \mathrm{~N}$ barrier (b), and band diagram comparison for MQW with GaN barrier and $\mathrm{In}_{0.05} \mathrm{Ga}_{0.95} \mathrm{~N}$ barrier (c).

Figure 5 Simulated radiative recombination rate in MQW with GaN barrier (sample A) and $\mathrm{In}_{0.05} \mathrm{Ga}_{0.95} \mathrm{~N}$ barrier (sample B). 

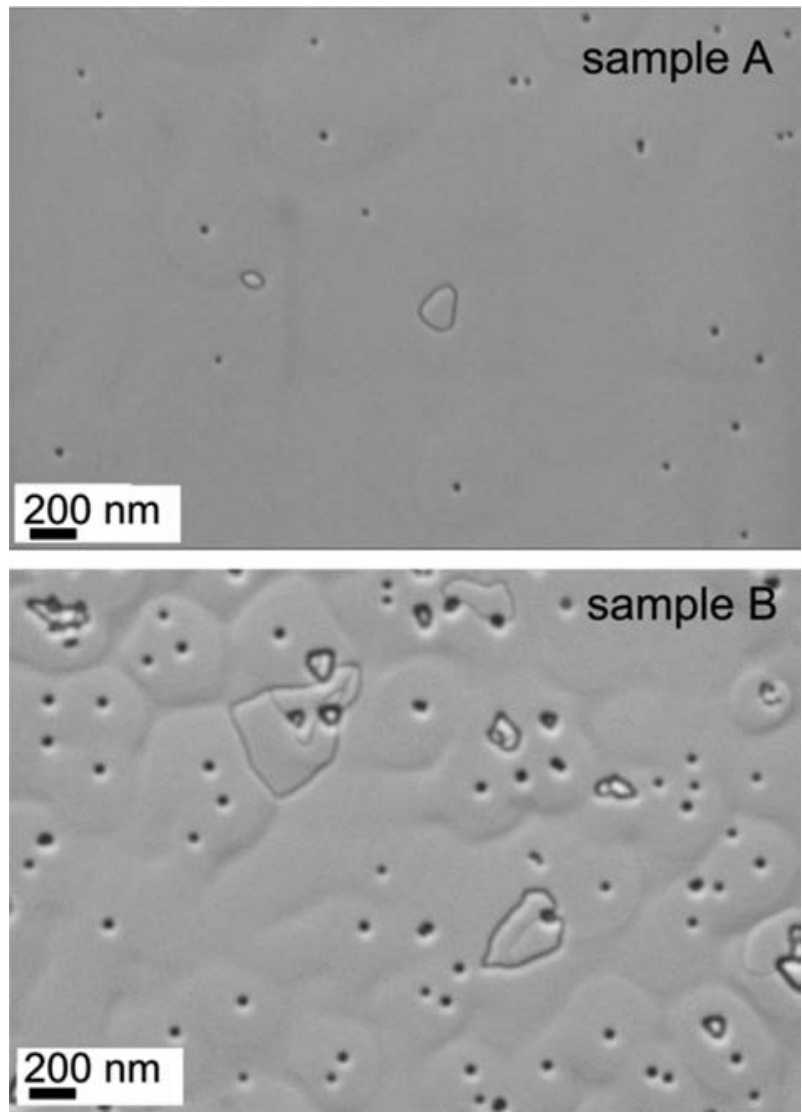

Figure 7 SEM surface morphology of MQW sample A with GaN barrier (top) and sample B with $\mathrm{In}_{0.05} \mathrm{Ga}_{0.95} \mathrm{~N}$ barrier (bottom).

barrier (sample A) and the sample with $\operatorname{In}_{0.05} \mathrm{Ga}_{0.95} \mathrm{~N}$ barrier (sample B) were carried out by SEM (Fig. 7).

It was observed that putting indium in the barrier caused degradation in the surface and an increase in the V-pit density and inclusion defects, however, the RMS roughness of the surface was still good and less than $2 \mathrm{~nm}$ for a $5 \times 5 \mu \mathrm{m}^{2}$ scan area, as observed in the AFM image (not shown here). However, a higher In content would increase the non-radiative recombination centers more and would not be the optimal content for obtaining the maximum IQE.

CL emission spectra for samples A and B are shown in Fig. 8. The emission intensity is doubled with the improved FWHM for InGaN barrier compared to those for $\mathrm{GaN}$ barrier, and the emission wavelength is blue shifted by $\sim 30 \mathrm{~nm}$. We attribute this blue shift to the increased effective bandgap, which we also observed in the theoretical simulation study. The peak around $550 \mathrm{~nm}$ is the defect yellow band because the spectrum is for room temperature CL. The In incorporation in wells for both of the samples was $18 \%$ as extracted from the fitting of the XRD $2 \theta-\omega$ scan data.

Low temperature (15 K) PL study was performed for the same sets of samples but with high In incorporation for the longer wavelength green-gap range emission.

The In content was $25 \%$ for both samples. The PL result is shown in Fig. 9. Similar result to the lower In content samples was observed, where a blue shift of $\sim 35 \mathrm{~nm}$ was

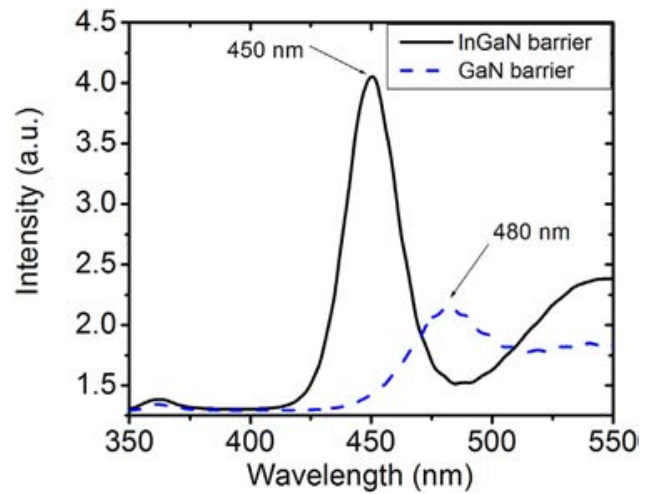

Figure 8 Room temperature cathodoluminescence of MQW sample A with $\mathrm{GaN}$ barrier and sample $\mathrm{B}$ with $\mathrm{In}_{0.05} \mathrm{Ga}_{0.95} \mathrm{~N}$ barrier.

observed with more than a threefold increase in the emission intensity and improved FWHM.

Though the InGaN barrier gives an improved emission and a higher IQE, the blue shift could cause a negative effect for getting longer wavelength emission in the green-gap range.

However, we have observed a red-shift of emission wavelength by using a semi-bulk (SB) $\mathrm{In}_{0.05} \mathrm{Ga}_{0.95} \mathrm{~N}$ buffer layer below the MQW which we attribute to the partial strain relaxation along with a slight compositional pulling, induced by a thickness increase of MQWs. A similar redshift result was reported in Ref. [19].

This SB buffer layer in combination with the InGaN barrier could compensate for the blue shift and can a give reasonably longer wavelength emission with higher emission intensity.

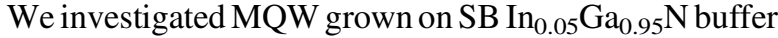
layer with $\mathrm{GaN}$ barrier (sample $\mathrm{C}$ ) and $\mathrm{In}_{0.05} \mathrm{Ga}_{0.95} \mathrm{~N}$ barrier (sample D). The In content was 14-15\%. A similar blue shift was observed for the sample with $\operatorname{In}_{0.05} \mathrm{Ga}_{0.95} \mathrm{~N}$ barrier (Fig. 10). The use of InGaN barrier increased the PL intensity by four times compared to the GaN barrier when both were grown on an SB buffer. This improvement, in addition to the effect of InGaN barrier discussed before, is also due to the fact that the InGaN SB buffer, which is unintentionally n-doped,

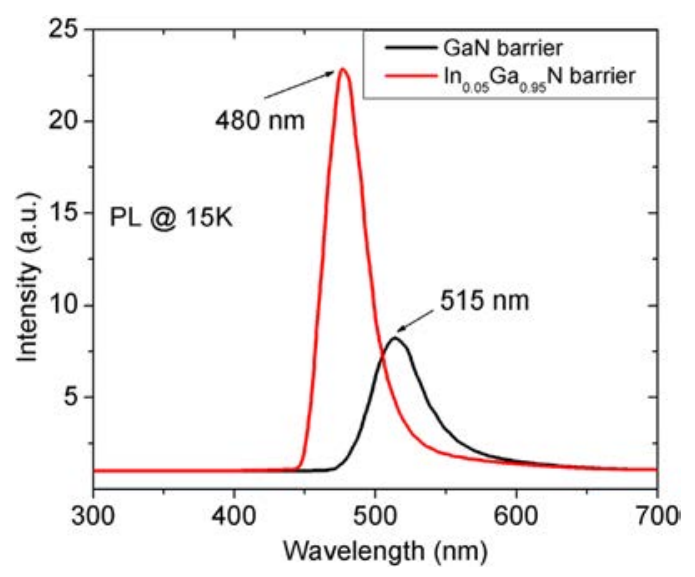

Figure 9 Low temperature photoluminescence of $25 \%$ In content MQW sample with GaN barrier and with $\mathrm{In}_{0.05} \mathrm{Ga}_{0.95} \mathrm{~N}$ barrier. 


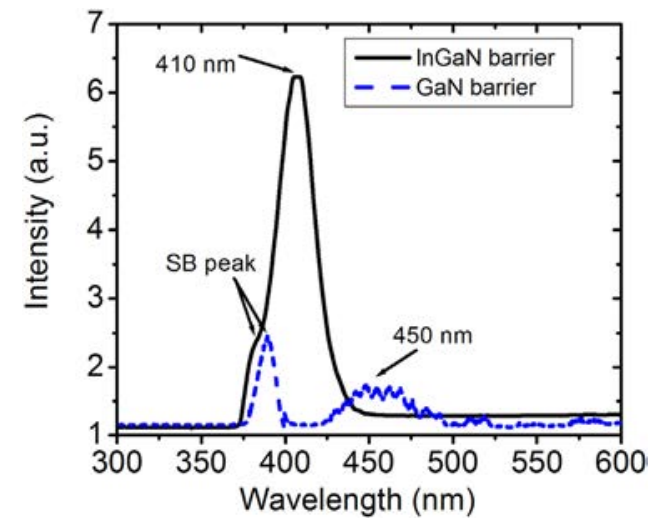

Figure 10 Low temperature $(77 \mathrm{~K})$ photoluminescence of MQW with $\mathrm{In}_{0.05} \mathrm{Ga}_{0.95} \mathrm{~N}$ barrier and with $\mathrm{GaN}$ barrier - both grown on a semi-bulk $\mathrm{In}_{0.05} \mathrm{Ga}_{0.95} \mathrm{~N}$ buffer.

acts as an electron reservoir and hence, can provide a higher amount of carriers and a much more homogeneous carrier distribution in the wells. However, a comparison between the two MQW structures, where both have an with InGaN barrier but one is without SB buffer layer and one is with such buffer, resulted in a net red-shift in the emission wavelength for the sample with the InGaN SB buffer. For longer wavelength emission, the simulation was carried out with an increased In content in the well and a proportionately scaled up In content in the barrier and SB InGaN buffer. The simulated room temperature IQE for different In contents in the well and in the barrier, and SB InGaN buffer for green emission is shown in Fig. 11. Though the efficiency at high current density decreases with In content higher than $25 \%$ in wells for conventional MQW, growing MQWs on SB InGaN buffer and with InGaN barrier with $20-25 \%$ In content, yields reduced droop at high current density with a theoretical IQE in the range of 50-60\% for the emission wavelength in the range of $\sim 530 \mathrm{~nm}$. We attribute this retention of high IQE, even with

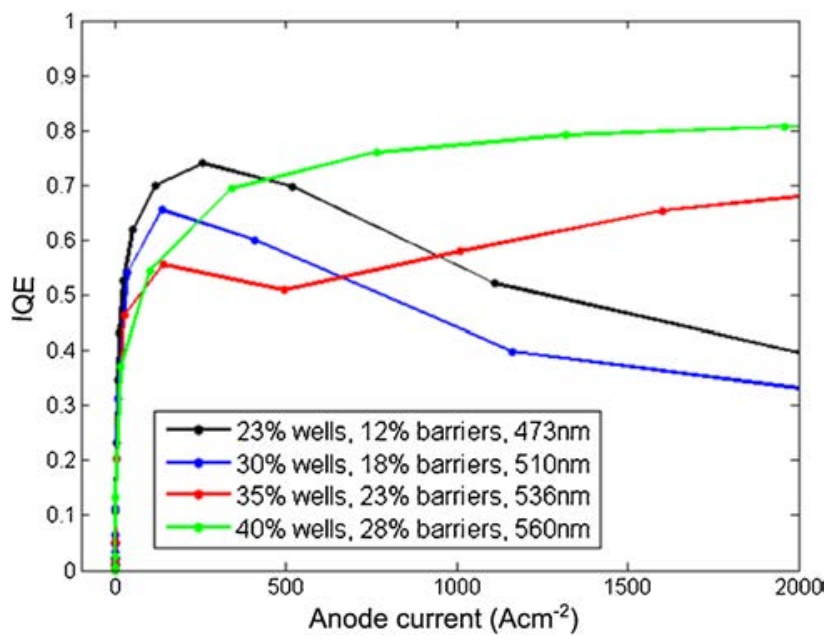

Figure 11 Simulated IQE for different In contents in well $\left(\operatorname{In}_{\mathrm{x}} \mathrm{Ga}_{1-\mathrm{x}} \mathrm{N}\right)$ and barrier $\left(\operatorname{In}_{\mathrm{y}} \mathrm{Ga}_{1-\mathrm{y}} \mathrm{N}\right)$ for $3 \mathrm{MQW}$ grown on semibulk $\operatorname{In}_{\mathrm{y}} \mathrm{Ga}_{1-\mathrm{y}} \mathrm{N}$ buffer. higher In in barrier, to the reduced droop at high current density which is a result of the reduced built-in electric field for using semi-bulk InGaN as buffer.

The realization of this longer wavelength green emission experimentally, by putting more indium in the well and in the barrier and buffer, is the subject of current study.

4 Conclusions We have demonstrated improvement in the IQE and the efficiency droop using $\mathrm{In}_{0.05} \mathrm{Ga}_{0.95} \mathrm{~N}$ barrier with $5 \%$ indium (In) in MQW that emits in the blue-to-green region. Both the simulation and the experiment show an increase in the emission intensity which is attributed to higher polarization match and a more homogeneous carrier distribution in the wells, that results in a higher overlap of the electron and hole wave function and increases the radiative recombination rate. However, putting more In than this threshold value in the barrier can deteriorate the crystal quality and reduce IQE by creating more nonradiative recombination centers. This InGaN barrier can be used for high efficiency longer wavelength LEDs, where the wavelength blue shift due to InGaN barrier can be compensated by the novel high quality semi-bulk InGaN buffer layer approach, and at the same time, the improvement of the emission intensity and the efficiency droop can be achieved utilizing the benefits of the InGaN barrier.

\section{References}

[1] J. Piprek, Phys. Status Solidi A 207, 2217-2225 (2010).

[2] T. Wunderer, M. Feneberg, F. Lipski, J. Wang, R. A. R. Leute, S. Schwaiger, K. Thonke, A. Chuvilin, U. Kaiser, S. Metzner, F. Bertram, J. Christen, G. J. Beirne, M. Jetter, P. Michler, L. Schade, C. Vierheilig, U. T. Schwarz, A. D. Dräger, A. Hangleiter, and F. Scholz, Phys. Status Solidi B 248, 549-560 (2011).

[3] Y. Yang, L. Zhang, T. Wei, and Y. Zeng, J. Display Technol. 11, 456-460 (2015).

[4] Y. K. Kuo, J. Y. Chang, M. C. Tsai, and S. H. Yen, Appl. Phys. Lett. 95, 011116 (2009).

[5] Y. M. Park, J. K. Son, H. J. Chung, C. Sone, and Y. Park, Appl. Phys. Lett. 95, 1-4 (2009).

[6] M.-H. Kim, M. F. Schubert, Q. Dai, J. K. Kim, E. F. Schubert, J. Piprek, and Y. Park, Appl. Phys. Lett. 91, 183507 (2007).

[7] M. F. Schubert, J. Xu, J. K. Kim, E. F. Schubert, M. H. Kim, S. Yoon, S. M. Lee, C. Sone, T. Sakong, and Y. Park, Appl. Phys. Lett. 93, 3-5 (2008).

[8] Y. Kuo, J. Chang, M. Tsai, S. Yen, Y. Kuo, J. Chang, M. Tsai, and S. Yen, Appl. Phys. Lett. 95, 011116 (2009).

[9] Y. Zhang, T. T. Kao, J. Liu, Z. Lochner, S. S. Kim, J. H. Ryou, R. D. Dupuis, and S. C. Shen, J. Appl. Phys. 109, 083115 (2011).

[10] Y. Yang and Y. Zeng, Phys. Status Solidi A 211, 1640-1644 (2014).

[11] Y. Yang and Y. Zeng, Opt. Commun. 326, 121-125 (2014).

[12] J. Xu, M. F. Schubert, A. N. Noemaun, D. Zhu, J. K. Kim, E. F. Schubert, M. H. Kim, H. J. Chung, S. Yoon, C. Sone, and Y. Park, Appl. Phys. Lett. 94, 215-218 (2009).

[13] Y. Yang and Y. Zeng, J. Appl. Phys. 117, 035705 (2015). 
[14] S. Gautier, C. Sartel, S. Ould-Saad, J. Martin, A. Sirenko, and A. Ougazzaden, J. Cryst. Growth. 298, 428-432 (2007).

[15] Y. El Gmili, G. Orsal, K. Pantzas, A. Ahaitouf, T. Moudakir, S. Gautier, G. Patriarche, D. Troadec, J. P. Salvestrini, and A. Ougazzaden, Opt. Mater. Express 3, 1111-1118 (2013).

[16] ATLAS User's Manual, Silvaco (2004).
[17] F. Bernardini, V. Fiorentini, and D. Vanderbilt, Phys. Rev. B 56, R10024(R) (1997).

[18] Z. Lin, H. Wang, Y. Lin, and M. Yang, J. Phys. D. Appl. Phys. 49, 115112 (2016).

[19] D. M. Van Den Broeck, D. Bharrat, A. M. Hosalli, N. A. El-Masry, and S. M. Bedair, Appl. Phys. Lett. 105, 31107 (2014). 